\title{
Prognosis Factors of Young Patients Undergoing Curative Resection for Hepatitis B Virus-Related Hepatocellular Carcinoma: A Multicenter Study
}

This article was published in the following Dove Press journal: Cancer Management and Research

\author{
Jianxing Zeng ${ }^{1-3, *}$ \\ Kongying Lin $\mathbb{D I D}^{4, *}$ \\ Huocheng Liu',* \\ Yao Huang $\mathbb{1}^{1-3}$ \\ Pengfei Guo 4 \\ Yongyi Zeng ${ }^{1-3}$ \\ Jinhua Zeng ${ }^{1-3}$ \\ Jingfeng Liu (D) ${ }^{1-3}$
}

'Department of Hepatic Surgery, Mengchao Hepatobiliary Hospital of Fujian Medical University, Fuzhou 350025,

People's Republic of China; ${ }^{2}$ The First Affiliated Hospital of Fujian Medical University, Fuzhou 350005, People's Republic of China; ${ }^{3}$ The Liver Center of Fujian Province, Fujian Medical University, Fuzhou 350025, People's Republic of China; ${ }^{4}$ Southeast Big Data Institute of Hepatobiliary Health, Mengchao Hepatobiliary Hospital of Fujian Medical University, Fuzhou 350025, People's Republic of China

*These authors contributed equally to this work
Correspondence: Jingfeng Liu Department of Hepatic Surgery, Mengchao Hepatobiliary Hospital of Fujian Medical University, Fuzhou 350025, People's Republic of China

$\mathrm{Tel} / \mathrm{Fax}+8659183705927$

Email drjingfeng@|26.com
Background: The prognosis of young and older patients with hepatocellular carcinoma (HCC) is controversial. We aim to compare the clinicopathological features and prognosis of young (age $\leq 40$ years) versus older patients (aged $>40$ years) with hepatitis B virus (HBV)related $\mathrm{HCC}$ after curative resection.

Methods: A total of 4504 patients with HBV-related HCC who underwent curative resection were included in this study and divided into young group $(n=699)$ and older group $(n=3805)$. Subgroup analyses were conducted to compare. Independent risk factors were identified by Cox regression analysis.

Results: Young patients had better ALBI grade, lower rates of liver cirrhosis, higher rates of elevated serum AFP levels, larger tumor size, higher rates of microvascular invasion and macrovascular invasion, higher rates of Edmondson grade III-IV, lower rates of tumor capsular, more advanced AJCC TNM stages and more advanced BCLC stages than older patients (All $\mathrm{p}<0.05)$. Meanwhile, young patients had a worse overall survival (OS) rate $(p=0.0091)$ and a worse recurrence-free survival (RFS) rate $(p=0.045)$ than older patients. Multivariate analysis revealed that AFP, resection margin, tumor size, tumor capsular, and macrovascular invasion were associated with OS. The independent risk factors associated with RFS were ALB, tumor size, microvascular invasion, and macrovascular invasion.

Conclusion: Young patients had better liver function, more aggressive tumor characteristics, and worse prognosis than older patients. A tumor size of $\geq 5 \mathrm{~cm}$ and macrovascular invasion were associated with poor OS and RFS in young patients. If tumors could be detected at the early stage by more frequent surveillance, long-term survival can be expected in the young patients.

Keywords: hepatocellular carcinoma, young, older, hepatectomy, prognosis

\section{Introduction}

Hepatocellular carcinoma (HCC) is the third leading cause of cancer-related death worldwide. ${ }^{1}$ Patients with hepatitis B virus (HBV) infection are one of the major risk factors for the development of $\mathrm{HCC}$, particularly those with chronic liver disease and cirrhosis. ${ }^{2}$ It is associated with $70-90 \%$ of patients with HCC in the highly endemic Asia-Pacific region, particularly in China. ${ }^{3}$ There are various kinds of treatments for HBV-related HCC, such as liver resection, liver transplantation, transarterial chemoembolization, radiofrequency ablation, etc. ${ }^{4}$ Liver resection is a mainstay of treatment for HBV-related HCC patients. ${ }^{5}$

With the development of modern medical technology and screening programs, HBV-related HCC is detected to be getting younger. ${ }^{2,6,7}$ However, a limited number 
of studies have reported the prognosis of young HBVrelated HCC patients and the conclusions are variable. Some authors have found that young patients have better long-term outcomes, ${ }^{8-10}$ whereas others have reported that young patients have advanced tumor factors, thereby indicating a poor prognosis. ${ }^{11,12}$ Several studies have demonstrated that the long-term outcomes after HCC resection are similar between young and older groups. ${ }^{13-15}$ Most of these studies enrolled less than 100 patients investigated the prognosis of young $\mathrm{HCC}$ patients. Hence, it remains controversial whether the prognosis of $\mathrm{HCC}$ in young patients is different from that in older patients.

To address this issue, we conducted a large multicenter study to compare the clinicopathological features and the prognosis of young patients (age $\leq 40$ years) versus older patients (aged $>40$ years) with HBV-related HCC after curative resection.

\section{Methods}

\section{Patients and Study Design}

This study was approved by the institutional ethics committee of Mengchao Hepatobiliary Hospital of Fujian Medical University (NO.:2019_069_01). Informed consent was obtained from each patient for their data to be used for research purposes. HBV-related HCC patients who underwent liver resection between January 2008 and December 2015 were extracted from primary liver cancer big data by an IT engineer, and then were verified by two researchers (Dr. Jianxing Zeng and Dr. Kongying Lin) in this study.

The inclusion criteria included: (1) 0 to 1 score of performance status, ${ }^{16}(2)$ positive hepatitis B surface antigen (HBsAg) and negative hepatitis $\mathrm{C}$ antibody, (3) no evidence of extrahepatic metastasis, (4) no history of preoperative anticancer treatment, (5) no history of other malignancies, and (6) curative resection with tumornegative resection margins (R0 resection). ${ }^{17}$ Patients who received palliative tumor resection, received recurrent tumor resection, had incomplete clinical data, died of severe surgical complications, and lost to follow-up within 60 days after discharge were excluded.

All patients received routine serological examination including white blood cell, platelet count, total bilirubin, albumin, alpha-fetoprotein (AFP), hepatitis B virus and hepatitis $\mathrm{C}$ virus immunology, and $\mathrm{HBV}$ deoxyribonucleic acid (HBV-DNA) load. Imaging studies included chest radiography, abdominal ultrasonography, and contrast- enhanced computed tomography (CT)/magnetic resonance imaging (MRI) of the abdomen.

Histopathological study of the resected specimens was performed independently by three pathologists who came to a consensus by discussion if there was any controversy. Histologic grading of HCC was based on the EdmondsonSteiner classification. ${ }^{18}$ The criterion of the American Association for the Study of Liver Diseases was used for pre-operative clinical diagnosis of HCC. ${ }^{19}$ All patients were staged using the American Joint Committee on Cancer (AJCC) staging system and Barcelona Clinic Liver Cancer (BCLC) staging system. ${ }^{20,21}$

\section{Follow-Up}

Patients were followed up once every 3 months for the first 2 years after discharge from hospitals and every 3-6 months in subsequent years. The follow-up program included liver function, AFP level, and abdominal ultrasound. Contrastenhanced CT or MRI was performed when tumor recurrence was clinically suspected. The diagnostic criteria for tumor recurrence were the same as for the initial diagnosis. The follow-up was censored on 31st October 2018.

The end-points of the study were overall survival (OS) and recurrence-free survival (RFS). OS was defined as the interval between the date of surgery and the date of patient death or the date of last follow-up. RFS was the interval between the date of surgery and the date when tumor recurrence was diagnosed or the date of patient death or the date of last follow-up.

\section{Statistical Analysis}

The age cut-off of 40 years was based on previous studies. $^{2,12,13,22-25}$ We defined young patients as aged $\leq 40$ years and older patients as aged $>40$ years. The clinicopathological features and the prognosis in young patients were compared with those in older patients.

Categorical variables were grouped on the basis of a normal reference value or clinical judgment. The albumin-bilirubin (ALBI) grade was calculated by the formula, $0.66 \times \log _{10}$ (bilirubin, $\left.\mu \mathrm{mol} / \mathrm{L}\right)-0.085 \times($ albumin, $\mathrm{g} / \mathrm{l}){ }^{26}$ According to previously described cut-off resulting in 2 grades: ALBI grade $1(\leq-2.60)$, grade $2(>-2.60$ to-1.39) and grade $3(>-1.39)$. ALBI grade 2 and ALBI grade 3 were group together due to the low sample size in the latter. The results were compared using the chi-square test or Fisher exact test. Continuous variables were compared using the Student's $t$-test or Mann-Whitney $U$-test for variables with an abnormal distribution. 
Kaplan-Meier method was used to estimate OS and RFS rates, and the difference between the two groups was analyzed by the Log rank test. Univariate and multivariate Cox proportional hazard regression was performed to detect the independent factors of OS and RFS. Subgroup analysis was conducted based on the univariate Cox model and the forest plot of subgroup analysis was depicted with each estimated HRs and 95\% CI. All statistical tests were 2-tailed and a p-value of less than 0.05 was considered statistically significant. All statistical analysis was performed with $\mathrm{R}$ version 3.5.2 (http://www.r-project.org)).

\section{Results}

\section{Comparison of Clinicopathological Features Between Young and Older HBV-Related HCC Patients}

During the study period, there were a total of 6028 patients with HBV-related HCC who received curative resection. 1524 patients were excluded because of extrahepatic metastasis $(n=207)$, preoperative anticancer treatment $(n=464)$, history of other malignancies $(n=56)$, palliative tumor resection $(n=174)$, recurrent tumor resection $(n=256)$, incomplete clinical data $(n=80)$, perioperative death $(n=33)$, and early lost to follow-up after discharge $(n=254)$. Finally, the study consisted of 4504 patients, which comprises 699 young HCC patients and 3805 older HCC patients. The flow chart of this patient selection is shown in Figure 1.

As summarized in Table 1, young patients had higher serum albumin levels $(\mathrm{p}<0.001)$, higher platelet counts $(\mathrm{p}<0.001)$, better ALBI grade $(\mathrm{p}<0.001)$, and lower rates of liver cirrhosis $(\mathrm{p}=0.001)$ than older patients. Considering tumor factors, young patients had higher rates of elevated serum AFP levels $(p<0.001)$, larger tumor size $(\mathrm{p}<0.001)$, higher rates of microvascular invasion $(p<0.001)$ and macrovascular invasion $(p<0.001)$, higher rates of Edmondson grade III-IV $(p=0.01)$, lower rates of tumor capsular $(\mathrm{p}=0.025)$, more advanced AJCC TNM stages $(\mathrm{p}<0.001)$ and more advanced BCLC stages $(p<0.001)$ than older patients.

\section{Comparison of Prognosis Between Young and Older HBV-Related HCC Patients}

The prognosis after liver resection for HBV-related HCC between young and older patients is shown in Figure 2. The overall survival rates at $1,3,5$ years were $81.2 \%$,

HBV related HCC patients who underwent hepatectomy between 2008 and 2015 were extracted from primary liver cancer big data $(n=6028)$

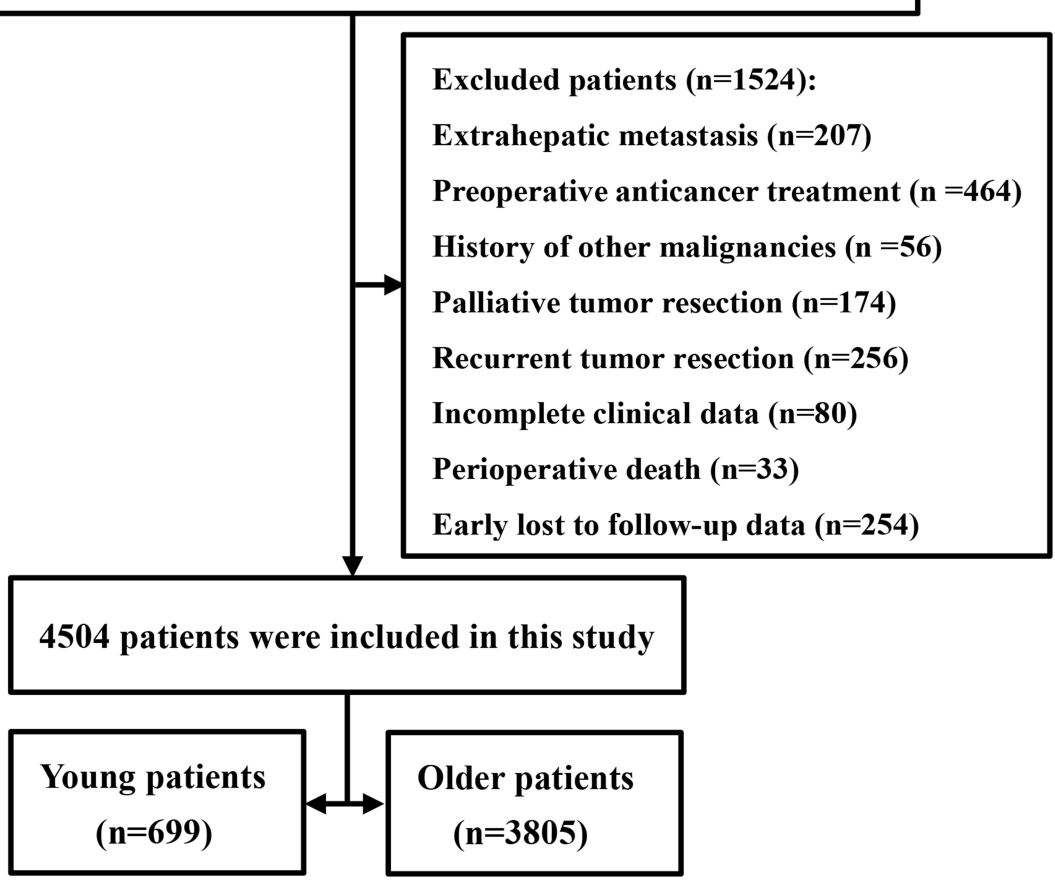

Figure I The flow chart of selected patients.

Abbreviations: $\mathrm{HBV}$, hepatitis B virus; $\mathrm{HCC}$, hepatocellular carcinoma. 
Table I Comparison of Clinicopathological Features Between Young and Older HBV-Related HCC Patients

\begin{tabular}{|c|c|c|c|}
\hline \multirow[t]{2}{*}{ Variables } & Young HCC & Older HCC & \multirow[t]{2}{*}{ p-value } \\
\hline & $(n=699)$ & $(n=3805)$ & \\
\hline Age, years, Median [IQR] & $36.0[32.0,39.0]$ & $53.0[47.0,60.0]$ & $<0.001$ \\
\hline $\begin{array}{c}\text { Gender } \\
\text { Female } \\
\text { Male }\end{array}$ & $\begin{array}{l}84(12.0 \%) \\
615(88.0 \%)\end{array}$ & $\begin{array}{l}519(13.6 \%) \\
3286(86.4 \%)\end{array}$ & 0.272 \\
\hline $\begin{array}{l}\text { AFP } \\
\quad<10 \mathrm{ng} / \mathrm{mL} \\
\geq 10 \mathrm{ng} / \mathrm{mL}\end{array}$ & $\begin{array}{l}|4|(20.2 \%) \\
558(79.8 \%)\end{array}$ & $\begin{array}{l}1153(30.3 \%) \\
2652(69.7 \%)\end{array}$ & $<0.001$ \\
\hline $\begin{array}{l}\text { HBV-DNA } \\
\quad<10001 \mathrm{~L} / \mathrm{L} \\
\geq 10001 \mathrm{~L} / \mathrm{L}\end{array}$ & $\begin{array}{l}79(11.3 \%) \\
620(88.7 \%)\end{array}$ & $\begin{array}{l}512(13.5 \%) \\
3293(86.5 \%)\end{array}$ & 0.136 \\
\hline $\begin{array}{l}\text { WBC, } 10^{9} / \mathrm{L} \text {, Mean (SD) } \\
\text { PLT, } 10^{9} / \mathrm{L}, \text { Mean (SD) } \\
\text { ALB, g/L, Mean (SD) } \\
\text { TBIL, } \mu \mathrm{mol} / \mathrm{L} \text {, Median } \\
\text { [IQR] }\end{array}$ & $\begin{array}{l}5.60(1.69) \\
182(70.4) \\
43.2(3.76) \\
13.4[10.5,17.4]\end{array}$ & $\begin{array}{l}5.33(1.79) \\
157(66.8) \\
41.8(3.61) \\
13.5[10.7,17.0]\end{array}$ & $\begin{array}{l}<0.001 \\
<0.001 \\
<0.001 \\
0.349\end{array}$ \\
\hline $\begin{array}{l}\text { ALBI grade } \\
\text { Grade I } \\
\text { Grade } 2\end{array}$ & $\begin{array}{l}597(85.4 \%) \\
102(14.6 \%)\end{array}$ & $\begin{array}{l}285 I(74.9 \%) \\
954(25.1 \%)\end{array}$ & $<0.001$ \\
\hline $\begin{array}{l}\text { Resection margin width } \\
<1 \mathrm{~cm} \\
\geq \mathrm{Icm}\end{array}$ & $\begin{array}{l}544(77.8 \%) \\
155(22.2 \%)\end{array}$ & $\begin{array}{l}3082(81.0 \%) \\
723(19.0 \%)\end{array}$ & 0.0582 \\
\hline $\begin{array}{l}\text { Blood transfusion } \\
\text { No } \\
\text { Yes }\end{array}$ & $\begin{array}{l}625(89.4 \%) \\
74(10.6 \%)\end{array}$ & $\begin{array}{l}3423(90.0 \%) \\
382(10.0 \%)\end{array}$ & 0.709 \\
\hline $\begin{array}{l}\text { Operative bleeding loss } \\
<800 \mathrm{~mL} \\
\geq 800 \mathrm{~mL}\end{array}$ & $\begin{array}{l}643(92.0 \%) \\
56(8.0 \%)\end{array}$ & $\begin{array}{l}3500(92.0 \%) \\
305(8.0 \%)\end{array}$ & 1.000 \\
\hline $\begin{array}{l}\text { Tumor size, cm Median } \\
\text { [IQR] }\end{array}$ & $5.80[3.70,9.20]$ & $5.00[3.30,8.00]$ & $<0.001$ \\
\hline $\begin{array}{l}\text { Tumor number } \\
\text { Solitary } \\
\text { Multiple }\end{array}$ & $\begin{array}{l}565(80.8 \%) \\
134(19.2 \%)\end{array}$ & $\begin{array}{l}3067(80.6 \%) \\
738(19.4 \%)\end{array}$ & 0.931 \\
\hline $\begin{array}{l}\text { Microvascular invasion } \\
\text { No } \\
\text { Yes }\end{array}$ & $\begin{array}{l}374(53.5 \%) \\
325(46.5 \%)\end{array}$ & $\begin{array}{l}2423(63.7 \%) \\
1382(36.3 \%)\end{array}$ & $<0.001$ \\
\hline $\begin{array}{l}\text { Macrovascular invasion } \\
\text { No } \\
\text { Yes }\end{array}$ & $\begin{array}{l}568(81.3 \%) \\
131(18.7 \%)\end{array}$ & $\begin{array}{l}3329(87.5 \%) \\
476(12.5 \%)\end{array}$ & $<0.001$ \\
\hline $\begin{array}{l}\text { Edmondson grade } \\
\text { I-II } \\
\text { III-IV }\end{array}$ & $\begin{array}{l}65(9.3 \%) \\
634(90.7 \%)\end{array}$ & $\begin{array}{l}490(12.9 \%) \\
3315(87.1 \%)\end{array}$ & 0.010 \\
\hline $\begin{array}{l}\text { Tumor capsular } \\
\text { No } \\
\text { Yes }\end{array}$ & $\begin{array}{l}163(23.3 \%) \\
536(76.7 \%)\end{array}$ & $\begin{array}{l}743(19.5 \%) \\
3062(80.5 \%)\end{array}$ & 0.025 \\
\hline
\end{tabular}

(Continued)
Table I (Continued)

\begin{tabular}{|l|l|l|l|}
\hline \multirow{2}{*}{ Variables } & Young HCC & Older HCC & \multirow{2}{*}{ p-value } \\
\cline { 2 - 3 } & $\mathbf{( n = 6 9 9 )}$ & $\mathbf{( n = 3 8 0 5 )}$ & \\
\hline $\begin{array}{l}\text { Satellite nodules } \\
\text { No }\end{array}$ & $395(56.5 \%)$ & $2258(59.3 \%)$ & \multirow{2}{*}{0.175} \\
Yes & $304(43.5 \%)$ & $1547(40.7 \%)$ & \\
\hline Liver cirrhosis & & & \\
No & $344(49.2 \%)$ & $1616(42.5 \%)$ & 0.001 \\
Yes & $355(50.8 \%)$ & $2189(57.5 \%)$ & \\
\hline BCLC stage & & & \\
O/A & $487(69.7 \%)$ & $2827(74.3 \%)$ & $<0.001$ \\
B & $81(11.6 \%)$ & $502(13.2 \%)$ & \\
C & $131(18.7 \%)$ & $476(12.5 \%)$ & \\
\hline AJCC TNM stage (8th) & & & \\
I & $323(46.2 \%)$ & $2057(54.1 \%)$ & $<0.001$ \\
II & $188(26.9 \%)$ & $97 \mid(25.5 \%)$ & \\
IIIA & $57(8.2 \%)$ & $301(7.9 \%)$ & \\
IIIB & $131(18.7 \%)$ & $476(12.5 \%)$ & \\
\hline
\end{tabular}

Notes: Categorical variables are presented as no. (\%). Mean (standard deviation) presented for normally distributed continuous variables, while median (interquartile range) was given to those with non-normally distributed continuous variables.

Abbreviations: HCC, hepatocellular carcinoma; AFP, alpha-fetoprotein; WBC, white blood cell; PLT, platelet count; ALB, albumin; TBIL, total bilirubin; ALBI grade, albumin-bilirubin grade; BCLC, Barcelona Clinic Liver Cancer; AJCC, American Joint Committee on Cancer; TNM, tumor, node, metastases; SD, standard deviation; IQR, interquartile range.

$61 \%$, and $45.3 \%$, respectively, in young patients with $\mathrm{HCC}$ and $86.4 \%, 65.3 \%$, and $47.7 \%$, respectively, in the older patients with HCC ( $\mathrm{p}=0.0091$, Figure $2 \mathrm{~A})$. The recurrencefree survival rates at $1,3,5$ years were $54.7 \%, 39.7 \%$, and $28.2 \%$, respectively, in young group and $63.8 \%, 41.1 \%$, and $28 \%$, respectively, in the older group $(\mathrm{p}=0.045$, Figure 2B).

To stratify by tumor factors and liver functional reserve, we performed several subgroup analyses of OS and RFS (Figures 3 and 4, respectively). After stratification by the BCLC staging system, there were no significant differences in OS and RFS between young and older groups in BCLC 0/A staging $(\mathrm{p}=0.17 ; \mathrm{p}=0.8)$ and BCLC $\mathrm{B}$ staging $(\mathrm{p}=0.26 ; \mathrm{p}=0.58$ ) (Figure $2 \mathrm{C}-\mathrm{F}$ ). In BCLC $\mathrm{C}$ staging, young $\mathrm{HCC}$ patients had a significantly worse prognosis than older HCC patients $(\mathrm{p}=0.018 ; \mathrm{p}=0.00017)$ (Figure $2 \mathrm{G}$ and $\mathrm{H}$ ).

\section{Risk Factors Associated with OS and RFS in Young HBV-Related HCC Patients}

The univariate and multivariate Cox analysis for determining the risk factors associated with OS and RFS in young HBV-related HCC patients are shown in Table 2. 
A

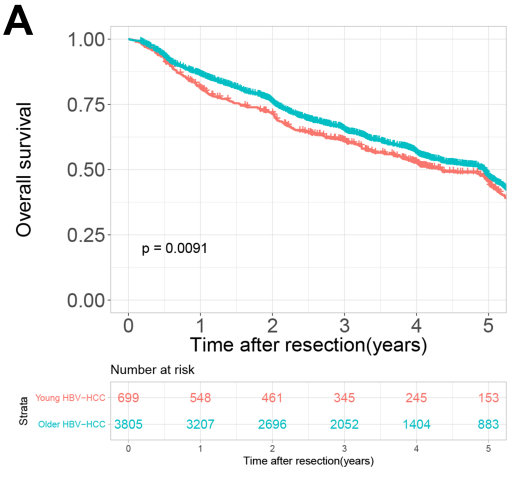

Strata - Young HBV-HCC - Older HBV-HCC

C

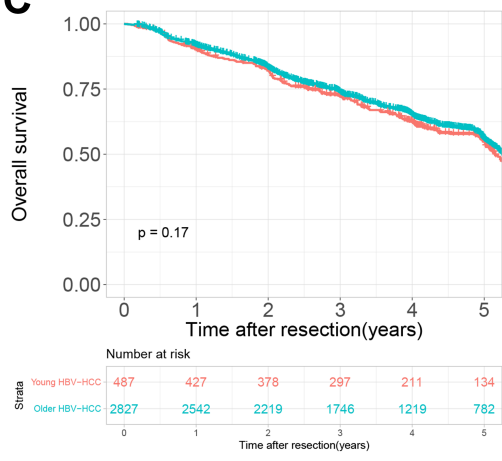

Strata - Young HBV-HCC - Older HBV-HCC

E

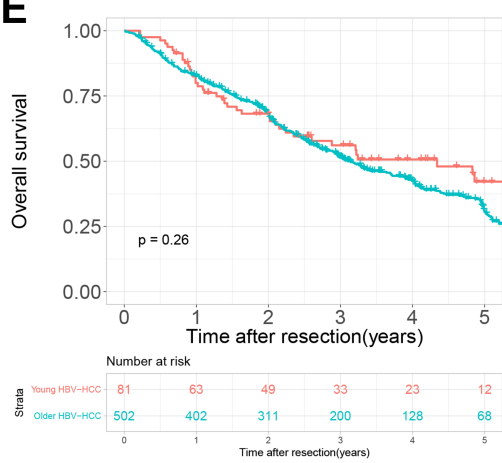

Strata - Young HBV-HCC - Older HBV-HCC

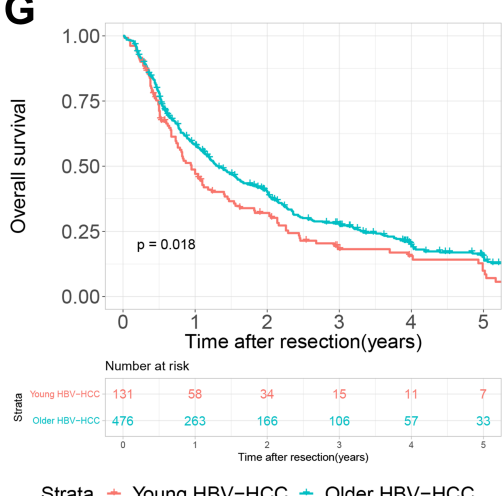

B

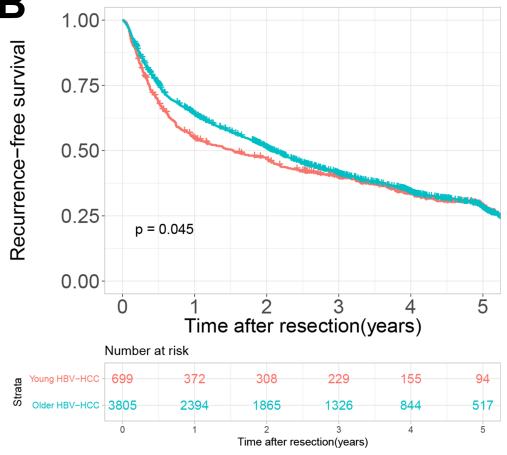

Strata - Young HBV-HCC - Older HBV-HCC

D

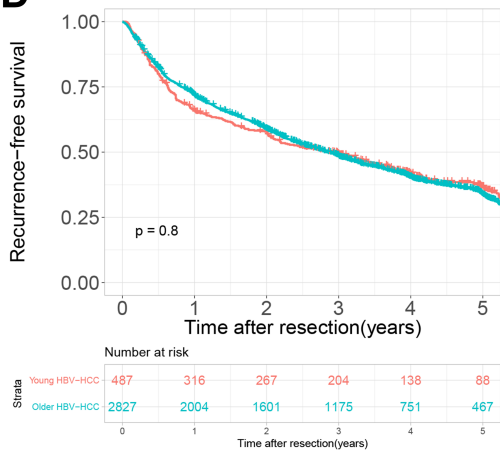

Strata - Young HBV-HCC - Older HBV-HCC

$\mathbf{F}$

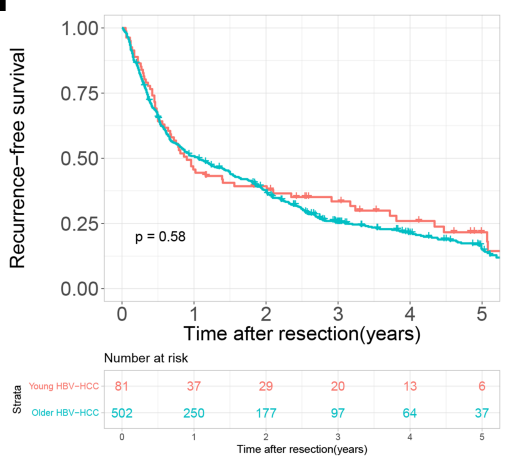

Strata - Young HBV-HCC - Older HBV-HCC

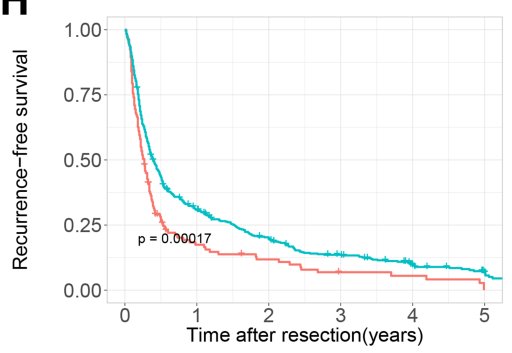

Number at risk

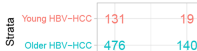

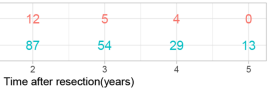

Strata - Young HBV-HCC - Older HBV-HCC

Figure 2 Comparison of prognosis between young and older groups. (A) OS in the entire patients; (B) RFS in the entire patients; (C) OS in BCLC 0/A staging; (D) RFS in BCLC 0/A staging; (E) OS in BCLC B staging; (F) RFS in BCLC B staging; (G) OS in BCLC C staging; (H) RFS in BCLC C staging.

Abbreviations: OS, overall survival; RFS, recurrence-free survival; BCLC, Barcelona Clinic Liver Cancer. 


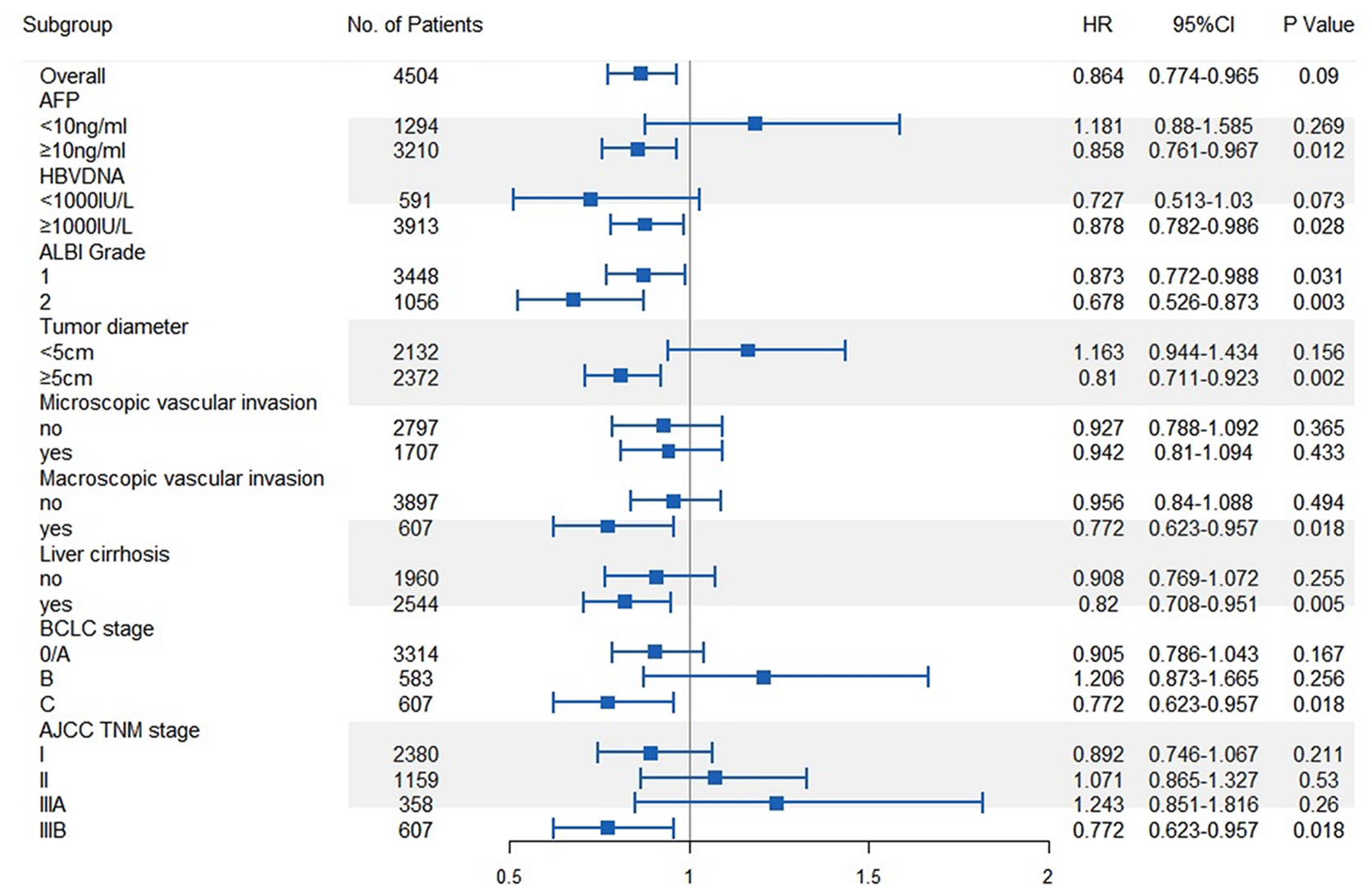

Figure 3 Subgroup analysis of overall survival between young and older groups.

Abbreviations: AFP, alpha-fetoprotein; ALBI grade, albumin-bilirubin grade; BCLC, Barcelona Clinic Liver Cancer; AJCC, American Joint Committee on Cancer; TNM, tumor, node, metastases; $\mathrm{HR}$, hazard ratio; $\mathrm{Cl}$, confidence interval.

Multivariate analysis revealed that a serum AFP levels of $\geq 10 \mathrm{ng} / \mathrm{mL}$, a resection margin width of $<1 \mathrm{~cm}$, a tumor size of $\geq 5 \mathrm{~cm}$, the absence of tumor capsular, and the presence of macrovascular invasion were the independent risk factors associated with mortality (Table 2). A serum ALB level of $<35 \mathrm{~g} / \mathrm{L}$, a tumor size of $\geq 5 \mathrm{~cm}$, the presence of microvascular invasion, and macrovascular invasion were the independent risk factors associated with tumor recurrence by multivariate analysis (Table 2).

\section{Discussion}

Generally, Hepatocellular carcinoma (HCC) usually occurs in middle-aged and elderly individuals. The age distribution of HCC varies according to geographical location and etiology. ${ }^{27}$ In areas where hepatitis B infection is endemic, screening programs for $\mathrm{HCC}$ in high-risk populations are important to increase the rate of early diagnosis and improve treatment outcome. ${ }^{2}$ Nonetheless, the prevalence of HBV-related HCC in young patients, especially in highrisk populations, has increased. ${ }^{3}$ However, the prognosis of young patients with HBV-related HCC is controversial.
Some authors have found that young patients have better long-term outcomes, ${ }^{8-10}$ whereas others have reported that young patients have advanced tumor factors, thereby indicating a poor prognosis. ${ }^{11,12}$ Several studies have demonstrated that the long-term outcomes after HCC resection are similar between the two groups. ${ }^{13-15}$

The variable conclusions may be attributed to the following two aspects. Firstly, the number of young and older patients with HCC was too small for accurate analysis in these studies. ${ }^{12,22-24}$ The present study focused on a relatively small proportion of young and older patients with HBV-related HCC in China, and this population was further restricted to patients who intended to curative hepatic resection with good liver functional reserve. Such strict patient selection could be fully compared the clinical characteristics, outcome, and prognostic factors after hepatectomy of young HCC patients with older HCC patients.

Secondly, the lack of standardization of what cut-off age constituted "young and older HCC". In some studies, the cut-off was defined as 30 or 40 years, whereas in others below 55 years. ${ }^{2,10,15,28}$ The determination of the 


\begin{tabular}{|c|c|c|c|c|c|}
\hline Subgroup & No. of Patients & & HR & $95 \% \mathrm{Cl}$ & $P$ Value \\
\hline $\begin{array}{l}\text { Overall } \\
\text { AFP }\end{array}$ & 4504 & & 0.907 & $0.824-0.998$ & 0.045 \\
\hline$<10 \mathrm{ng} / \mathrm{ml}$ & 1294 & & 0.993 & $0.797-1.238$ & 0.954 \\
\hline$\geq 10 \mathrm{ng} / \mathrm{ml}$ & 3210 & & 0.928 & $0.834-1.032$ & 0.167 \\
\hline \multicolumn{6}{|l|}{ HBVDNA } \\
\hline$<1000 I U / L$ & 591 & & 0.695 & $0.517-0.935$ & 0.016 \\
\hline$\geq 1000 I U / L$ & 3913 & & 0.936 & $0.847-1.036$ & 0.201 \\
\hline \multicolumn{6}{|l|}{ ALBI Grade } \\
\hline 1 & 3448 & & 0.895 & $0.805-0.995$ & 0.039 \\
\hline 2 & 1056 & & 0.804 & $0.64-1.009$ & 0.059 \\
\hline \multicolumn{6}{|l|}{ Tumor diameter } \\
\hline$<5 \mathrm{~cm}$ & 2132 & $\longrightarrow$ & 1.19 & $1.007-1.406$ & 0.042 \\
\hline $\begin{array}{l}\geq 5 \mathrm{~cm} \\
\text { Microsconic vascular invasion }\end{array}$ & 2372 & & 0.831 & $0.739-0.934$ & 0.002 \\
\hline no & 2797 & & 1.039 & $0.907-1.191$ & 0.579 \\
\hline yes & 1707 & & 0.861 & $0.752-0.985$ & 0.029 \\
\hline \multicolumn{6}{|l|}{ Macroscopic vascular invasion } \\
\hline no & 3897 & & 1 & $0.897-1.115$ & 0.996 \\
\hline yes & 607 & & 0.677 & $0.552-0.83$ & $<0.001$ \\
\hline \multicolumn{6}{|l|}{ Liver cirrhosis } \\
\hline no & $\longmapsto$ & & 0.841 & $0.731-0.968$ & 0.016 \\
\hline \multicolumn{6}{|l|}{$\mathrm{BCLC}$ stage } \\
\hline $0 / \mathrm{A}$ & 3314 & -1 & 0.985 & $0.874-1.109$ & 0.801 \\
\hline B & 583 & -1 & 1.078 & $0.827-1.405$ & 0.58 \\
\hline $\mathrm{C}$ & 607 & & 0.677 & $0.552-0.83$ & $<0.001$ \\
\hline \multicolumn{5}{|l|}{ AJCC TNM stage } & 0.599 \\
\hline ॥ & 1159 & , & 0.928 & $0.774-1.112$ & 0.418 \\
\hline IIIA & 358 & & 1.328 & $0.961-1.836$ & 0.086 \\
\hline \multirow[t]{2}{*}{ IIIB } & $\longmapsto-1$ & & 0.677 & $0.552-0.83$ & $<0.001$ \\
\hline & 0.5 & 1.5 & & & \\
\hline
\end{tabular}

Figure 4 Subgroup analysis of recurrence-free survival between young and older groups.

Abbreviations: AFP, alpha-fetoprotein; ALBI grade, albumin-bilirubin grade; BCLC, Barcelona Clinic Liver Cancer; AJCC, American Joint Committee on Cancer; TNM, tumor, node, metastases; $\mathrm{HR}$, hazard ratio; $\mathrm{Cl}$, confidence interval.

cut-off age for older HCC patients has also varied in the literature. $^{28-32}$ We defined young patients as aged $\leq 40$ years and older patients as aged $>40$ years. The age cutoff of 40 years was based on previous studies. ${ }^{2,10,11,20-23}$
We believe this allows for more meaningful comparisons with other studies. Besides, the Chinese guidelines for the management of $\mathrm{HCC}$ recommend screening of males beginning at age $40 . .^{33}$ Hence, we were interested in

Table 2 Univariate and Multivariate Cox Regression for OS and RFS in Young HBV Relate HCC Patients

\begin{tabular}{|c|c|c|c|c|c|c|c|c|}
\hline \multirow[t]{3}{*}{ Variables } & \multicolumn{4}{|l|}{ os } & \multicolumn{4}{|l|}{ RFS } \\
\hline & \multicolumn{2}{|l|}{ Univariate } & \multicolumn{2}{|l|}{ Multivariate } & \multicolumn{2}{|l|}{ Univariate } & \multicolumn{2}{|l|}{ Multivariate } \\
\hline & HR (95\% Cl) & p-value & HR (95\% Cl) & p-value & HR (95\% Cl) & p-value & HR $(95 \% \mathrm{Cl})$ & p-value \\
\hline \multicolumn{9}{|c|}{ Gender (Female as ref) } \\
\hline Male & $1.24(0.90-1.70)$ & 0.19 & & & I.0I (0.99-I.03) & 0.37 & & \\
\hline \multicolumn{9}{|c|}{$\operatorname{AFP}(<10 \mathrm{ng} / \mathrm{mL}$ as ref $)$} \\
\hline$\geq 10 \mathrm{ng} / \mathrm{mL}$ & $2.15(1.59-2.90)$ & $<0.01$ & $1.48(1.09-2.02)$ & 0.01 & $1.56(1.24-1.97)$ & $<0.01$ & & \\
\hline \multicolumn{9}{|c|}{ HBV-DNA $(<1000 I U / L$ as ref $)$} \\
\hline$\geq 10001 \mathrm{U} / \mathrm{L}$ & $0.84(0.59-1.18)$ & 0.30 & & & $0.94(0.70-1.25)$ & 0.65 & & \\
\hline \multicolumn{9}{|c|}{ WBC $\left(<4 \times 10^{9} / L\right.$ as ref $)$} \\
\hline$\geq 4 \times 10^{9} / \mathrm{L}$ & $1.20(0.90-1.59)$ & 0.22 & & & $1.35(1.05-1.74)$ & 0.02 & & \\
\hline
\end{tabular}

(Continued) 
Table 2 (Continued).

\begin{tabular}{|c|c|c|c|c|c|c|c|c|}
\hline \multirow[t]{3}{*}{ Variables } & \multicolumn{4}{|l|}{ os } & \multicolumn{4}{|l|}{ RFS } \\
\hline & \multicolumn{2}{|l|}{ Univariate } & \multicolumn{2}{|l|}{ Multivariate } & \multicolumn{2}{|l|}{ Univariate } & \multicolumn{2}{|l|}{ Multivariate } \\
\hline & HR (95\% Cl) & p-value & HR (95\% Cl) & p-value & HR (95\% Cl) & p-value & HR $(95 \% \mathrm{CI})$ & p-value \\
\hline \multicolumn{9}{|c|}{ PLT $\left(<100 \times 10^{9} / \mathrm{L}\right.$ as ref $)$} \\
\hline$\geq 100 \times 10^{9} / \mathrm{L}$ & $1.29(0.91-1.83)$ & 0.15 & & & $1.54(1.12-2.12)$ & $<0.01$ & & \\
\hline \multicolumn{9}{|c|}{ ALB $(<35 \mathrm{~g} / \mathrm{L}$ as ref) } \\
\hline$\geq 35 \mathrm{~g} / \mathrm{L}$ & $0.62(0.30-1.26)$ & 0.18 & & & $0.45(0.25-0.83)$ & 0.01 & $0.39(0.20-0.75)$ & $<0.01$ \\
\hline \multicolumn{9}{|c|}{ TBIL $(<17.1 \mu \mathrm{mol} / \mathrm{L}$ as ref) } \\
\hline$\geq 17.1 \mu \mathrm{mol} / \mathrm{L}$ & $1.24(0.99-1.54)$ & 0.06 & & & $1.13(0.93-1.38)$ & 0.21 & & \\
\hline \multicolumn{9}{|c|}{ ALBI grade (Grade I as ref) } \\
\hline Grade 2 & $1.63(1.25-2.12)$ & $<0.01$ & & & $1.41(1.11-1.78)$ & $<0.01$ & & \\
\hline \multicolumn{9}{|c|}{ Resection margin width $(<1 \mathrm{~cm}$ as ref) } \\
\hline$\geq 1 \mathrm{~cm}$ & $0.56(0.43-0.72)$ & $<0.01$ & $0.66(0.50-0.87)$ & $<0.01$ & $0.69(0.55-0.85)$ & $<0.01$ & & \\
\hline \multicolumn{9}{|c|}{ Blood transfusion (no as ref) } \\
\hline Yes & $2.67(2.02-3.53)$ & $<0.01$ & & & $2.24(1.73-2.90)$ & $<0.01$ & & \\
\hline \multicolumn{9}{|c|}{ Operative bleeding loss ( $<800 \mathrm{~mL}$ as ref) } \\
\hline$\geq 800 \mathrm{~mL}$ & $2.41(1.77-3.29)$ & $<0.01$ & & & $1.74(1.29-2.35)$ & $<0.01$ & & \\
\hline \multicolumn{9}{|c|}{ Tumor size $(<5 \mathrm{~cm}$ as ref) } \\
\hline$\geq 5 \mathrm{~cm}$ & $3.08(2.44-3.87)$ & $<0.01$ & $2.10(1.63-2.70)$ & $<0.01$ & $2.64(2.18-3.20)$ & $<0.01$ & $2.04(1.65-2.50)$ & $<0.01$ \\
\hline \multicolumn{9}{|c|}{ Tumor number (Solitary as ref) } \\
\hline Multiple & $1.55(1.21-1.97)$ & $<0.01$ & & & $1.59(1.28-1.97)$ & $<0.01$ & & \\
\hline \multicolumn{9}{|c|}{ Microvascular invasion (no as ref) } \\
\hline Yes & $2.21(\mid .80-2.7 I)$ & $<0.01$ & & & $2.25(1.88-2.69)$ & $<0.01$ & $1.50(1.22-1.84)$ & $<0.01$ \\
\hline \multicolumn{9}{|c|}{ Macrovascular invasion (no as ref) } \\
\hline Yes & $4.28(3.40-5.38)$ & $<0.01$ & $2.29(1.75-3.00)$ & $<0.01$ & $4.35(3.5 \mathrm{I}-5.40)$ & $<0.01$ & $2.77(2.15-3.58)$ & $<0.01$ \\
\hline \multicolumn{9}{|c|}{ Edmondson grade (I-II as ref) } \\
\hline III-IV & $2.86(1.78-4.59)$ & $<0.01$ & & & $2.19(1.53-3.14)$ & $<0.01$ & & \\
\hline \multicolumn{9}{|c|}{ Tumor capsular (no as ref) } \\
\hline Yes & $0.5 \mathrm{I}(0.4 \mathrm{I}-0.64)$ & $<0.01$ & $0.69(0.55-0.87)$ & $<0.01$ & $0.60(0.49-0.73)$ & $<0.01$ & & \\
\hline \multicolumn{9}{|c|}{ Satellite nodules (no as ref) } \\
\hline Yes & $1.76(1.44-2.16)$ & $<0.01$ & & & $1.52(1.27-1.81)$ & $<0.01$ & & \\
\hline \multicolumn{9}{|c|}{ Liver cirrhosis (no as ref) } \\
\hline Yes & $1.20(0.98-1.47)$ & 0.08 & & & $1.08(0.9 \mid-1.29)$ & 0.38 & & \\
\hline
\end{tabular}

Abbreviations: OS, overall survival; RFS, recurrence-free survival; AFP, alpha-fetoprotein; WBC, white blood cell; PLT, platelet count; ALB, albumin; TBIL, total bilirubin; ALBI grade, albumin-bilirubin grade; $\mathrm{HR}$, hazard ratio; $\mathrm{Cl}$, confidence interval; Ref, reference. 
evaluating the prognosis in HBV-related HCC patients below this age threshold.

In the present study, the results demonstrated that young patients had higher serum AFP levels, larger tumor size, higher rates of microvascular invasion and macrovascular invasion, higher rates of Edmondson grade III-IV, lower rates of tumor capsular, more advanced AJCC TNM stages, and more advanced BCLC stages than older patents. Meanwhile, young patients also had worse OS and RFS rate than older patients, which is consistent with previous study. ${ }^{11,12}$

Multivariate analysis revealed that a tumor size of $\geq 5 \mathrm{~cm}$ and macrovascular invasion were associated with OS and RFS in young patients, so aggressive tumor factors lead to poor prognosis in young patients. Previous studies have shown young patients diagnosed with HCC more often had symptomatic presentations and were less likely to be identified by surveillance. ${ }^{2,14}$ It implies that if tumors could be detected at the early stage by more frequent surveillance and radical treatment is performed, longterm survival can be expected in the young patients.

\section{Conclusions}

This multicenter study indicated that young patients had better liver function, more aggressive tumor characteristics, and worse prognosis than older patients. A tumor size of $\geq 5 \mathrm{~cm}$ and macrovascular invasion lead to poor prognosis in young patients.

\section{Abbreviations}

HCC, hepatocellular carcinoma; AFP, alpha-fetoprotein; HBV, hepatitis B virus; WBC, white blood cell; PLT, platelet count; ALB, albumin; TBIL, total bilirubin; ALBI, albumin-bilirubin; BCLC, Barcelona Clinic Liver Cancer; AJCC, American Joint Committee on Cancer; TNM, tumor, node, metastases; SD, standard deviation; $\mathrm{IQR}$, interquartile range.

\section{Data Sharing Statement}

The datasets used and/or analyzed during the current study are available from the corresponding author on reasonable request.

\section{Ethics Approval and Informed Consent}

This study was approved by the institutional ethics committee of Mengchao Hepatobiliary Hospital of Fujian
Medical University. Informed consent was obtained from each patient for their data to be used for research purposes.

\section{Acknowledgments}

The authors thank Zhenwei Chen and ChuanChun Chen for their help in data collection, and Dr. Zhiqing Liu for his assistance with the statistical analysis of this study.

\section{Funding}

This study was supported by the Special Fund of Fujian Development and Reform Commission (31010308), the Natural Science Foundation of Fujian Province (2018J01140), and the Key Clinical Specialty Discipline Construction Program of Fuzhou (201912002).

\section{Disclosure}

The authors declare no conflicts of interest.

\section{References}

1. Torre LA, Bray F, Siegel RL, Ferlay J, Lortet-Tieulent J, Jemal A. Global cancer statistics, 2012. CA Cancer J Clin. 2015;65(2):87-108. doi:10.3322/caac. 21262

2. Lam CM, Chan AO, Ho P, et al. Different presentation of hepatitis B-related hepatocellular carcinoma in a cohort of 1863 young and old patients - implications for screening. Aliment Pharmacol Ther. 2004;19(7):771-777. doi:10.1111/j.1365-2036.2004.01912.x

3. Ishikawa T. Clinical features of hepatitis B virus-related hepatocellular carcinoma. World J Gastroenterol. 2010;16(20):2463-2467. doi:10.3748/wjg.v16.i20.2463

4. Llovet JM, Zucman-Rossi J, Pikarsky E, et al. Hepatocellular carcinoma. Nat Rev Dis Primers. 2016;2:16018. doi:10.1038/ $\operatorname{nrdp} .2016 .18$

5. Omata M, Lesmana LA, Tateishi R, et al. Asian Pacific Association for the Study of the Liver consensus recommendations on hepatocellular carcinoma. Hepatol Int. 2010;4(2):439-474.

6. Kim JH, Choi MS, Lee H, et al. Clinical features and prognosis of hepatocellular carcinoma in young patients from a hepatitis B-endemic area. J Gastroenterol Hepatol. 2006;21(3):588-594. doi:10.1111/j.1440-1746.2005.04127.x

7. Sezaki H, Kobayashi M, Hosaka T, et al. Hepatocellular carcinoma in noncirrhotic young adult patients with chronic hepatitis B viral infection. $J$ Gastroenterol. 2004;39(6):550-556. doi:10.1007/ s00535-003-1341-2

8. Nathan H, Schulick RD, Choti MA, Pawlik TM. Predictors of survival after resection of early hepatocellular carcinoma. Ann Surg. 2009;249(5):799-805. doi:10.1097/SLA.0b013e3181a38eb5

9. Hung HH, Chiou YY, Hsia CY, et al. Survival rates are comparable after radiofrequency ablation or surgery in patients with small hepatocellular carcinomas. Clin Gastroenterol Hepatol. 2011;9(1):79-86. doi:10.1016/j.cgh.2010.08.018

10. Lee CR, Lim JH, Kim SH, et al. A comparative analysis of hepatocellular carcinoma after hepatic resection in young versus elderly patients. J Gastrointest Surg. 2012;16(9):1736-1743. doi:10.1007/ s11605-012-1966-7

11. Cho SJ, Yoon JH, Hwang SS, Lee HS. Do young hepatocellular carcinoma patients with relatively good liver function have poorer outcomes than elderly patients? J Gastroenterol Hepatol. 2007;22 (8):1226-1231. doi:10.1111/j.1440-1746.2007.04914.x 
12. Ha SY, Sohn I, Hwang SH, Yang JW, Park CK. The prognosis of hepatocellular carcinoma after curative hepatectomy in young patients. Oncotarget. 2015;6(21):18664-18673. doi:10.18632/oncotarget.4330

13. Chen $\mathrm{CH}$, Chang TT, Cheng KS, et al. Do young hepatocellular carcinoma patients have worse prognosis? The paradox of age as a prognostic factor in the survival of hepatocellular carcinoma patients. Liver Int. 2006;26(7):766-773. doi:10.1111/j.14783231.2006.01309.x

14. Chan AC, Poon RT, Ng KK, Lo CM, Fan ST, Wong J. Changing paradigm in the management of hepatocellular carcinoma improves the survival benefit of early detection by screening. Ann Surg. 2008;247(4):666-673. doi:10.1097/SLA.0b013e31816a747a

15. Su CW, Lei HJ, Chau GY, et al. The effect of age on the long-term prognosis of patients with hepatocellular carcinoma after resection surgery: a propensity score matching analysis. Arch Surg. 2012;147 (2):137-144. doi:10.1001/archsurg.2011.288

16. Llovet JM, Di Bisceglie AM, Bruix J, et al. Design and endpoints of clinical trials in hepatocellular carcinoma. J Natl Cancer Inst. 2008;100(10):698-711. doi:10.1093/jnci/djn134

17. Zhang XP, Gao YZ, Chen ZH, et al. An Eastern Hepatobiliary Surgery Hospital/Portal Vein Tumor thrombus scoring system as an aid to decision making on hepatectomy for hepatocellular carcinoma patients with portal vein tumor thrombus: a multicenter study. Hepatology. 2019;69(5):2076-2090. doi:10.1002/hep.30490

18. Zhou L, Rui J-A, Zhou W-X, Wang S-B, Chen S-G, Qu Q. Edmondson-Steiner grade: a crucial predictor of recurrence and survival in hepatocellular carcinoma without microvascular invasion. Pathol Res Pract. 2017;213(7):824-830. doi:10.1016/j. prp.2017.03.002

19. Marrero JA, Kulik LM, Sirlin CB, et al. Diagnosis, staging, and management of hepatocellular carcinoma: 2018 practice guidance by the American Association for the Study of Liver Diseases. Hepatology. 2018;68(2):723-750. doi:10.1002/hep.29913

20. Chun YS, Pawlik TM, Vauthey JN. 8th edition of the AJCC cancer staging manual: pancreas and hepatobiliary cancers. Ann Surg Oncol. 2018;25(4):845-847. doi:10.1245/s10434-017-6025-x

21. Galle PR, Forner A, Llovet JM, European Association for the Study of the Liver. Electronic address eee, European Association for the Study of the L. EASL clinical practice guidelines: management of hepatocellular carcinoma. $J$ Hepatol. 2018;69(1):182-236. doi:10.1016/j.jhep.2018.03.019

22. Yamazaki Y, Kakizaki S, Sohara N, et al. Hepatocellular carcinoma in young adults: the clinical characteristics, prognosis, and findings of a patient survival analysis. Dig Dis Sci. 2007;52(4):1103-1107. doi:10.1007/s10620-006-9578-2
23. Shimada S, Kamiyama T, Yokoo H, et al. Clinicopathological characteristics and prognostic factors in young patients after hepatectomy for hepatocellular carcinoma. World J Surg Oncol. 2013;11:52. doi:10.1186/1477-7819-11-52

24. Chang PE, Ong WC, Lui HF, Tan CK. Is the prognosis of young patients with hepatocellular carcinoma poorer than the prognosis of older patients? A comparative analysis of clinical characteristics, prognostic features, and survival outcome. $J$ Gastroenterol. 2008;43 (11):881-888. doi:10.1007/s00535-008-2238-x

25. Niederle IM, Worns MA, Koch S, et al. Clinicopathologic features and prognosis of young patients with hepatocellular carcinoma in a large German cohort. J Clin Gastroenterol. 2012;46(9):775-778. doi:10.1097/MCG.0b013e31826102cc

26. Johnson PJ, Berhane S, Kagebayashi C, et al. Assessment of liver function in patients with hepatocellular carcinoma: a new evidence-based approach-the ALBI grade. J Clin Oncol. 2015;33 (6):550-558. doi:10.1200/JCO.2014.57.9151

27. Bosch FX, Ribes J, Diaz M, Cleries R. Primary liver cancer: worldwide incidence and trends. Gastroenterology. 2004;127(5 Suppl 1): S5-S16. doi:10.1053/j.gastro.2004.09.011

28. Shen J, Li C, Yan L, et al. Short- and long-term outcomes between young and older HCC patients exceeding the milan criteria after hepatectomy. Ann Hepatol. 2018;17(1):134-143. doi:10.5604/ 01.3001.0010.7545

29. Saneto H, Kobayashi M, Kawamura Y, et al. Clinicopathological features, background liver disease, and survival analysis of HCV-positive patients with hepatocellular carcinoma: differences between young and elderly patients. $J$ Gastroenterol. 2008;43 (12):975-981. doi:10.1007/s00535-008-2268-4

30. Tandon P, Garcia-Tsao G. Prognostic indicators in hepatocellular carcinoma: a systematic review of 72 studies. Liver Int. 2009;29 (4):502-510. doi:10.1111/j.1478-3231.2008.01957.x

31. Kaibori M, Yoshii K, Yokota I, et al. Impact of advanced age on survival in patients undergoing resection of hepatocellular carcinoma: report of a Japanese Nationwide Survey. Ann Surg. 2019;269 (4):692-699. doi:10.1097/SLA.0000000000002526

32. Hung AK, Guy J. Hepatocellular carcinoma in the elderly: meta-analysis and systematic literature review. World J Gastroenterol. 2015;21(42):12197-12210. doi:10.3748/wjg.v21. i42.12197

33. Zhou J, Sun HC, Wang Z, et al. Guidelines for diagnosis and treatment of primary liver cancer in China (2017 edition). Liver Cancer. 2018;7(3):235-260. doi:10.1159/000488035

\section{Publish your work in this journal}

Cancer Management and Research is an international, peer-reviewed open access journal focusing on cancer research and the optimal use of preventative and integrated treatment interventions to achieve improved outcomes, enhanced survival and quality of life for the cancer patient.
The manuscript management system is completely online and includes a very quick and fair peer-review system, which is all easy to use. Visit http://www.dovepress.com/testimonials.php to read real quotes from published authors. 